Research Article

\title{
A Loop-Line Antenna with Enhanced Bandwidth of Circular Polarization
}

\author{
Kazuhide Hirose $\mathbb{D D}^{1}{ }^{1}$ Motoshi Nakatsu, ${ }^{1}$ and Hisamatsu Nakano ${ }^{2}$ \\ ${ }^{1}$ College of Engineering, Shibaura Institute of Technology, Tokyo 135-8548, Japan \\ ${ }^{2}$ Science and Engineering, Hosei University, Tokyo 184-8584, Japan \\ Correspondence should be addressed to Kazuhide Hirose; khirose@sic.shibaura-it.ac.jp
}

Received 17 November 2019; Accepted 1 February 2020; Published 26 February 2020

Academic Editor: Ping Li

Copyright (c) 2020 Kazuhide Hirose et al. This is an open access article distributed under the Creative Commons Attribution License, which permits unrestricted use, distribution, and reproduction in any medium, provided the original work is properly cited.

\begin{abstract}
We investigate a circularly polarized $(\mathrm{CP})$ transmission-line antenna composed of loop radiation elements, called a loop-line antenna. To enhance the CP wave bandwidth, an isolated loop radiation element is first analyzed using the method of moments. It is found that a $\mathrm{CP}$ wave bandwidth for a $3 \mathrm{~dB}$ axial-ratio criterion has a maximum value of $8 \%$. On the basis of the analysis results, we design the loop-line antenna. It is found that the antenna shows a $\mathrm{CP}$ wave bandwidth of $6 \%$, which is twice as wide as that of a conventional loop-line antenna. The analysis results are verified with experimental ones. In addition, a condition for wideband $\mathrm{CP}$ radiation is discussed and found to be a composite radiation element consisting of two CP loops.
\end{abstract}

\section{Introduction}

A transmission-line antenna (line antenna) is in a class with a series-fed linear array and can radiate a circularly polarized (CP) wave $[1,2]$. The antenna is characterized by a simple feed system in comparison with that of a conventional array, since the feed line itself contributes to $\mathrm{CP}$ wave radiation. So far, the line antennas have been designed using several kinds of radiation elements above a ground plane, such as rampart [3], crank [4], and loop elements [5, 6].

A recent study using a loop element has shown that the $\mathrm{CP}$ wave bandwidth of a line antenna can be increased [7]. This has become possible with a study of an isolated loop element without an array environment. The loop height above the ground plane has been selected for wideband $\mathrm{CP}$ radiation.

This paper is an extended version of the preliminary conference one [7] written by the present authors. The study of the isolated loop element is newly focused on the reason why the wideband CP radiation is obtained. For this, a more in-depth analysis than the preliminary one [7] is performed in Section 3 using the method of moments [8]. Even in Section 2 showing a need for selection of loop height above the ground plane, we add new results (shown with dotted lines in Figures 1 and 2), which play an important role of finding a condition for wideband CP radiation in Section 3.

In Section 4, we replace all the simulated results of the line antenna in [7] with new ones that can be validated by experimental work. This is based on an evolution of the antenna design, by which input impedance matching a $50-\Omega$ coaxial line is realized without deterioration in the $\mathrm{CP}$ radiation. Note that there have been only simulated results in [7], since the input impedance that satisfies a VSWR of less than 2 has yet to be researched.

\section{Loop Elements}

Figure 3 shows a loop antenna located at height $h$ above a ground plane. The antenna is composed of a circular loop of circumference $C$, parallel wires of distance $d$ [6], and a vertical wire $F-F^{\prime}$ of length $h$, whose bottom end $F^{\prime}$ is excited by a coaxial line, as shown in Figure 3(c).

At each value of loop height $h$ ranging from $\lambda_{0} / 10$ to $\lambda_{0} / 4$, we appropriately choose the loop parameters $(C, d)$ for the antenna to radiate a $\mathrm{CP}$ wave using the method of moments [8], where $\lambda_{0}$ is the free-space wavelength at a test frequency 


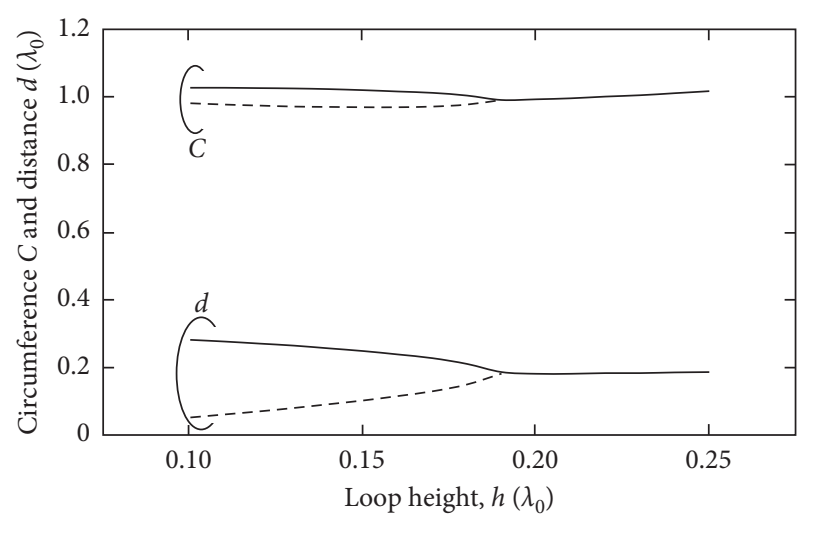

$$
\left\{\begin{array}{l}
\text { - Loop } \# 1 \bigcirc \\
--- \text { Loop } \# 2 \bigcirc
\end{array}\right.
$$

Figure 1: Simulated configuration values of two loops \#1 and 2 optimized for CP radiation versus loop height above the ground plane. Note that the dotted lines vanish for $h \geq 0.18 \lambda_{0}$, since there exists only one loop.

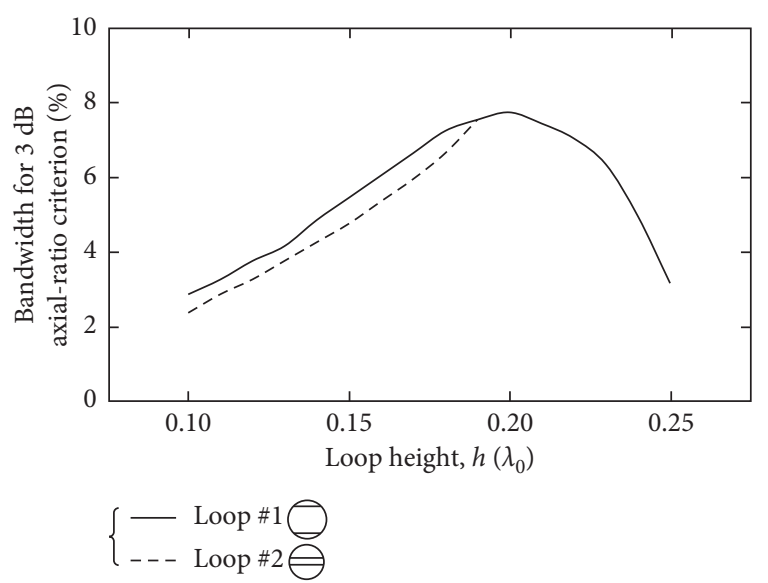

Figure 2: Simulated CP wave bandwidths of two loops \#1 and 2 versus loop height above the ground plane. Note that the dotted line vanishes for $h \geq 0.18 \lambda_{0}$, since there exists only one loop, as shown in Figure 1.

$f_{0}$. The wire radius is the same $\left(\rho=\lambda_{0} / 200\right)$ as that in [6]. Note that the ground plane is assumed to be of infinite extent and image theory is used in the analysis.

Figure 1 shows the simulated values of $(C, d)$ that are optimized for CP radiation versus loop height $h$. A loop antenna having these configuration values can radiate a $\mathrm{CP}$ wave with an axial ratio of less than $3 \mathrm{~dB}$. It is observed that as the loop height increases from $h=0.10 \lambda_{0}$, the difference between solid and dotted lines becomes small and vanishes at $h=0.18 \lambda_{0}$. This means that when $h<0.18 \lambda_{0}$, there exist two CP loops having different configuration values from each other at the same loop height $h$; one has larger $C$ and larger $d$ (see the solid lines) than the other, as shown in the inset and Figures 3(a) and 3(b).

The simulated CP wave bandwidths as a function of loop height $h$ are shown in Figure 2. The bandwidth is evaluated for a $3 \mathrm{~dB}$ axial-ratio criterion. It is found that the bandwidths of the two CP loops for $h<0.18 \lambda_{0}$ increase with an increase in the loop height $h$. A maximum bandwidth of $7.8 \%$ is obtained at $h=0.20 \lambda_{0}$, where there exists only one loop. Note that the dotted line (implying existence of the two CP loops mentioned in Figure 1) vanishes at $h=0.18 \lambda_{0}$.

The simulated radiation pattern of the antenna with $h=0.20 \lambda_{0}$ is shown in Figure $4(\mathrm{a})$. The pattern is illustrated with right- $\left(E_{\mathrm{R}}\right)$ and left-hand $\left(E_{\mathrm{L}}\right) \mathrm{CP}$ wave components. It is seen that a CP beam is formed in a direction normal to the antenna plane, in the $+z$-axis direction. The half-power beam width (HPBW) is $82^{\circ}$ and the gain is $7.3 \mathrm{~dB}$. For comparison, the radiation pattern of a conventional antenna with $h=0.125 \lambda_{0}$ [6] is also shown in Figure 4(b), where the HPBW and gain are $70^{\circ}$ and $9.1 \mathrm{~dB}$, respectively. The HPBW of the present antenna with $h=0.20 \lambda_{0}$ is wider than that of the conventional one by $12^{\circ}$, resulting in the lower gain.

\section{Modified Loop Elements}

So far, we have found a loop antenna showing wideband CP radiation. Subsequently, we analyze modified loop antennas to know a condition for the wideband $\mathrm{CP}$ radiation.

Figure 5 shows the modified antennas considered here. They are created from the present antenna shown in Figure 3; one has a loop without a chord $\beta-\gamma$ shown in Figure 5(a) and the other has a loop without two arcs of $\alpha-F$ and $\beta-\gamma$ shown in Figure 5(b). Note that the modified antennas are still specified by two parameters, loop circumference $C$, and parallel-wire distance $d$, as shown in Figure 5.

The simulated CP wave bandwidths versus loop height $h$ are shown in Figure 6. It is found that both the modified antennas can radiate a CP wave with an axial ratio of less than $3 \mathrm{~dB}$. It is also found that each modified antenna does not show wideband $C P$ radiation in comparison with the present antenna, as shown in Figure 2. This fact means that wideband CP radiation is obtained for a composite (present) antenna consisting of the two CP-modified loops, since both the modified loops are created from the present antenna, as mentioned in Figure 5 (also recall that there exist the two CP loops when $h<0.18 \lambda_{0}$, as shown in Figures 1 and 2 ). It can be said that a composite antenna consisting of two CP loops is a condition for the wideband CP radiation found in Section 2.

\section{Loop-Line Antenna}

In Sections 2 and 3, we have discussed wideband CP radiation which is attributed to a composite element consisting of two CP loops. Using this composite element, we design a line antenna to enhance the CP wave bandwidth.

Figure 7 shows the line antenna located at height $h$ above a ground plane. The antenna is composed of $N$ loop elements and connecting lines of length $L$, and excited at the left end $F$ using a coaxial line. A tapered feed $F-F^{\prime}$ is adopted for input impedance matching a $50-\Omega$ coaxial line, as shown in Figure $7(\mathrm{~b})$. The tapered-feed configuration is specified by $\left(l_{1}, l_{2}\right.$, and $\left.l_{3}\right)$. Note that the antenna is made of wires of radius $\rho[4-6]$.

We design the line antenna to radiate a CP broadside beam (in the $+z$-axis direction). The beam direction and 


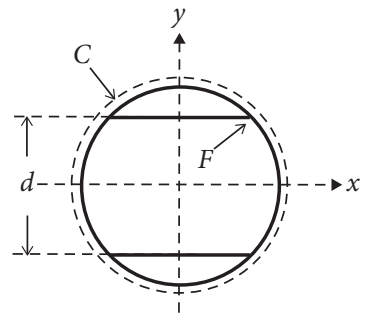

(a)

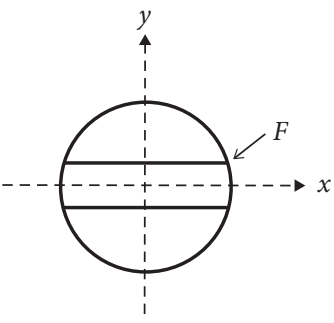

(b)

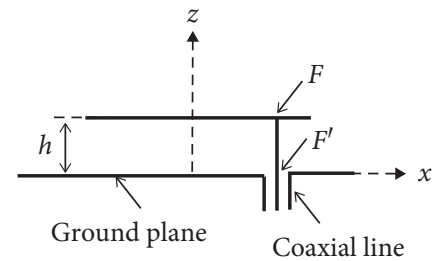

(c)

FIgUre 3: Two circularly polarized loop elements of \# 1 and 2. (a) Top view of a loop \#1, which has larger circumference $C$ and larger distance $d$ than a loop \#2. (b) Top view of the loop \#2. (c) Side view.

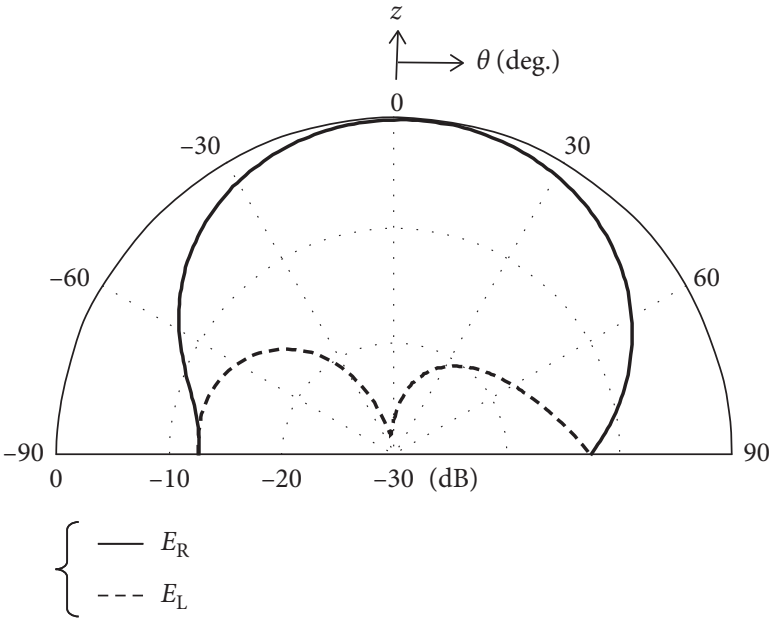

(a)

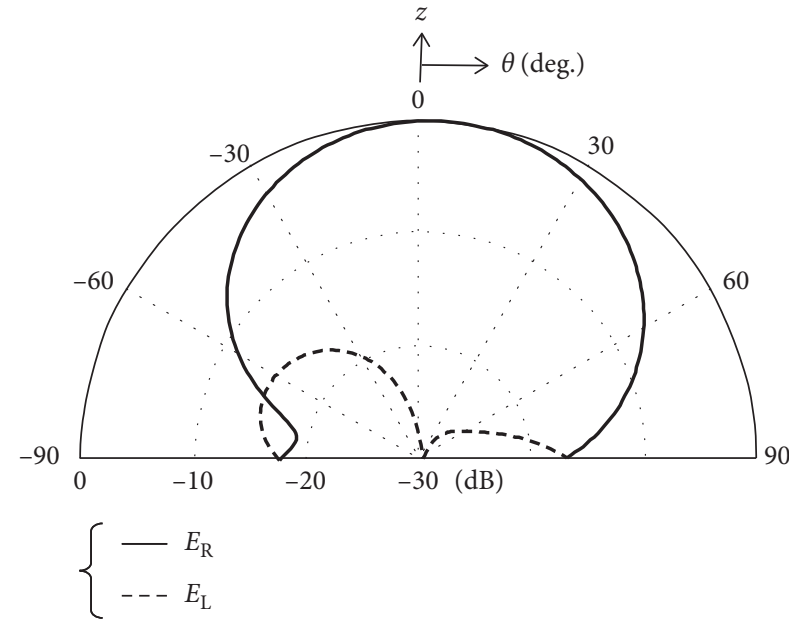

(b)

Figure 4: Simulated radiation patterns in $\phi=0^{\circ}$ plane. (a) Present loop element with $h=0.20 \lambda_{0}$. (b) Conventional loop element with $h=0.125 \lambda_{0}[6]$.

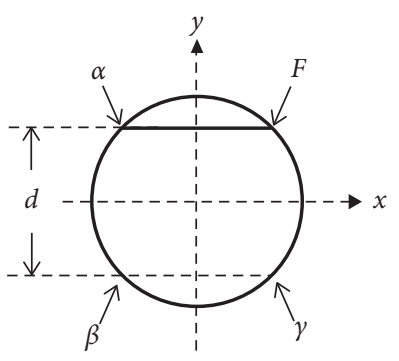

(a)

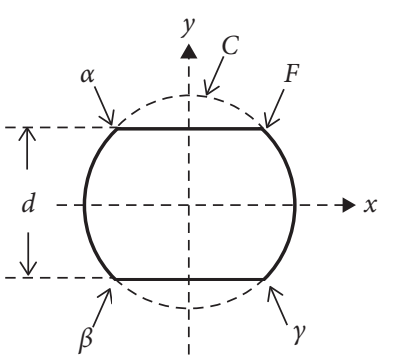

(b)

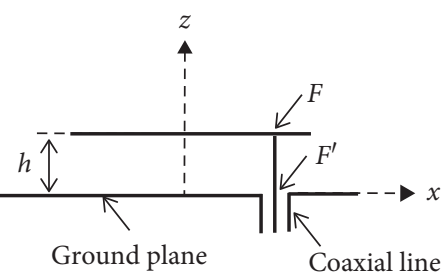

(c)

Figure 5: Two circularly polarized, modified loop elements that are created from the loop element, as shown in Figure 3. (a) Top view of a modified loop \#1 without a chord $\beta-\gamma$. (b) Top view of a modified loop \#2 without two arcs of $\alpha-F$ and $\beta-\gamma$. (c) Side view.

axial ratio are adjusted with the connecting-line length $L$ and loop parameters $(C, d)$, respectively. We take the antenna height to be $h=0.20 \lambda_{0}$, where each loop element should show the wideband CP radiation discussed in Section 2. The other configuration parameters are fixed to be the same values as those in [6]: $(\rho, N)=\left(\lambda_{0} / 200,4\right)$ to facilitate comparison.

We take the following two steps to efficiently determine the parameters:
(1) First, the tapered feed $F-F^{\prime}$ is changed to a direct feed, as shown in Figure $7(\mathrm{c})$, where a straight wire $F-F^{\prime}$ is used [7]. The line-antenna parameters $(L, C, d)$ are determined for the $\mathrm{CP}$ broadside beam. The determined values of $(L, C, d)$ are fixed in the second step.

(2) The direct feed is replaced with the tapered feed $F-F^{\prime}$. The tapered-feed parameters $\left(l_{1}, l_{2}, l_{3}\right)$ are determined for a VSWR of less than 2 under the condition that the $\mathrm{CP}$ broadside beam in the first step does not deteriorate. 


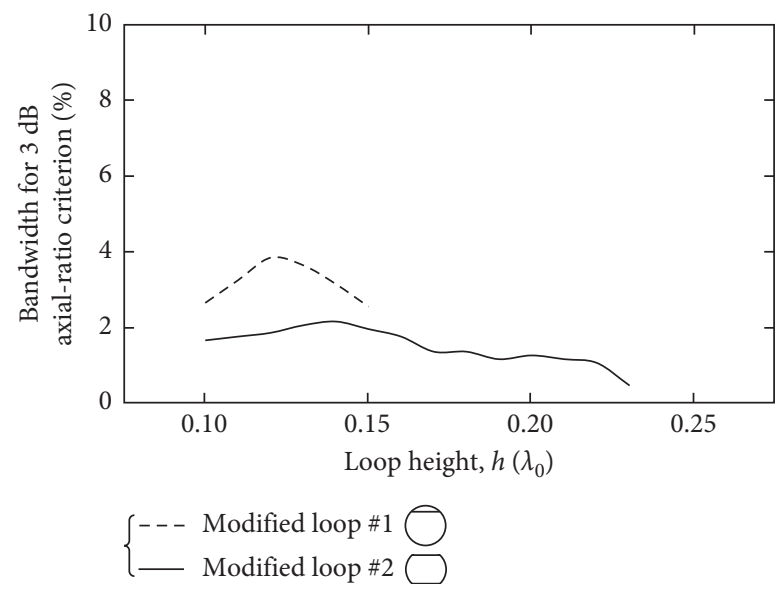

FIgURE 6: Simulated CP wave bandwidths of two modified loops \#1 and 2 versus loop height above the ground plane.

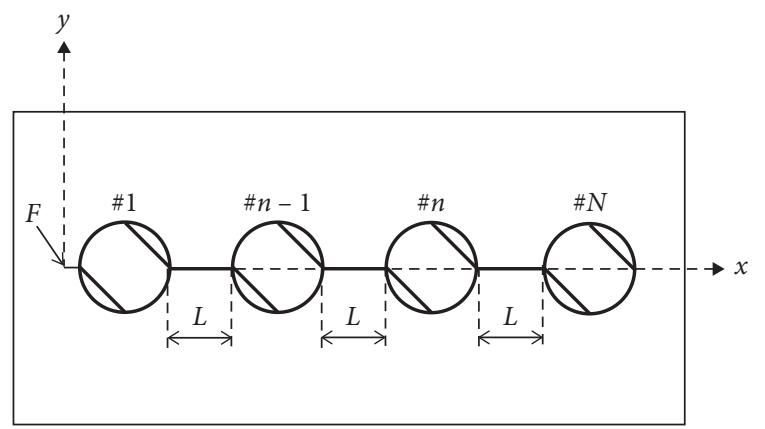

(a)

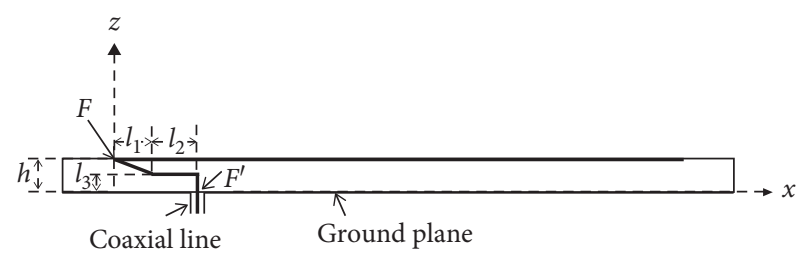

(b)

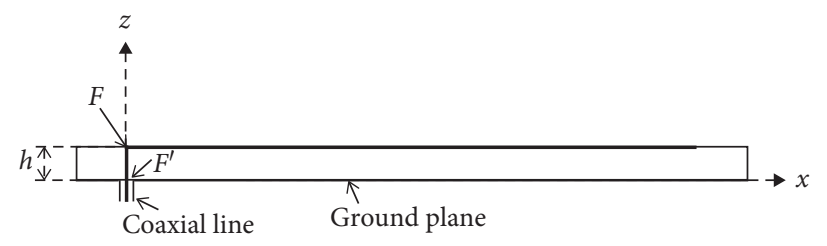

(c)

Figure 7: Loop-line antenna. (a) Top view. (b) Side view with tapered feed $F-F^{\prime}$. (c) Side view with direct feed $F-F^{\prime}$ [7].

Calculations in the first step show that the $\mathrm{CP}$ broadside beam is obtained for line-antenna parameters of $(L, C$, $d)=\left(0.53 \lambda_{0}, 1.01 \lambda_{0}, 0.26 \lambda_{0}\right)$. Further calculations in the second step reveal that a VSWR of less than 2 is obtained for taperedfeed parameters of $\left(l_{1}, l_{2}, l_{3}\right)=\left(0.25 \lambda_{0}, 0.17 \lambda_{0}, 0.02 \lambda_{0}\right)$.

The abovementioned values of the configuration parameters are summarized in Table 1, together with those of a conventional line antenna [6]. There is a difference between the values of height $h$, which is a key parameter for enhancing the CP wave bandwidth, as discussed in Section 2. Also, recall that the present and conventional line antennas have the same values of $(\rho, N)$.

The simulated radiation pattern is shown with solid and dotted lines in Figure 8(a). It is observed that the antenna radiates a $\mathrm{CP}$ beam in the $+z$-axis direction. The HPBW is $13^{\circ}$ and the gain is $14.1 \mathrm{~dB}$. For comparison, the simulated radiation pattern of the conventional line antenna with $h=0.125 \lambda_{0}$ [6] is also shown in Figure 8(b), where the HPBW and gain are $13^{\circ}$ and $14.5 \mathrm{~dB}$, respectively.

Solid lines in Figure 9(a) show the simulated axial ratio and gain in the $+z$-axis direction versus frequency. It is found that the axial ratio remains less than $3 \mathrm{~dB}$ in a frequency bandwidth of $6.1 \%$, where the gain is more than $14 \mathrm{~dB}$. For comparison, dotted lines show those of the conventional line antenna [6]. The CP wave bandwidth is $3.4 \%$. It should be emphasized that the bandwidth of the present line antenna is wider than that of the conventional 
TABLE 1: Values of antenna configuration parameters.

Loop-line antenna Height $h$ Wire radius $\rho$ Number of loops $N$ Line-antenna parameters $(L, C, d)$ Tapered-feed parameters $\left(l_{1}, l_{2}, l_{3}\right)$

\begin{tabular}{llllll}
\hline Present & $1 / 5$ & $1 / 200$ & 4 & $(0.53,1.01,0.26)$ & $(0.25,0.17,0.02)$ \\
Conventional [6] & $1 / 8$ & $1 / 200$ & 4 & $(0.37,1.02,0.29)$ & $(0.04,0.19,0.11)$
\end{tabular}

Unit: $\lambda_{0}$ other than $N$.

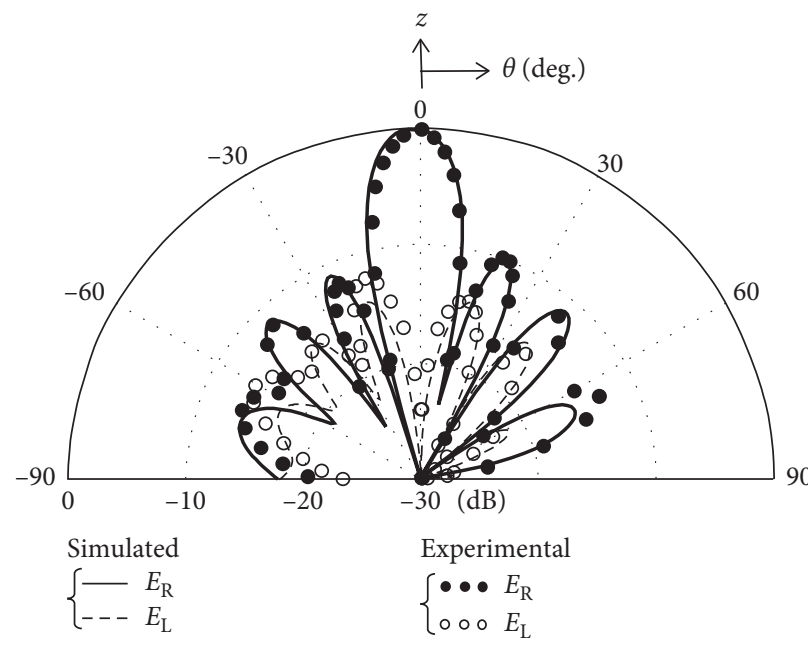

(a)

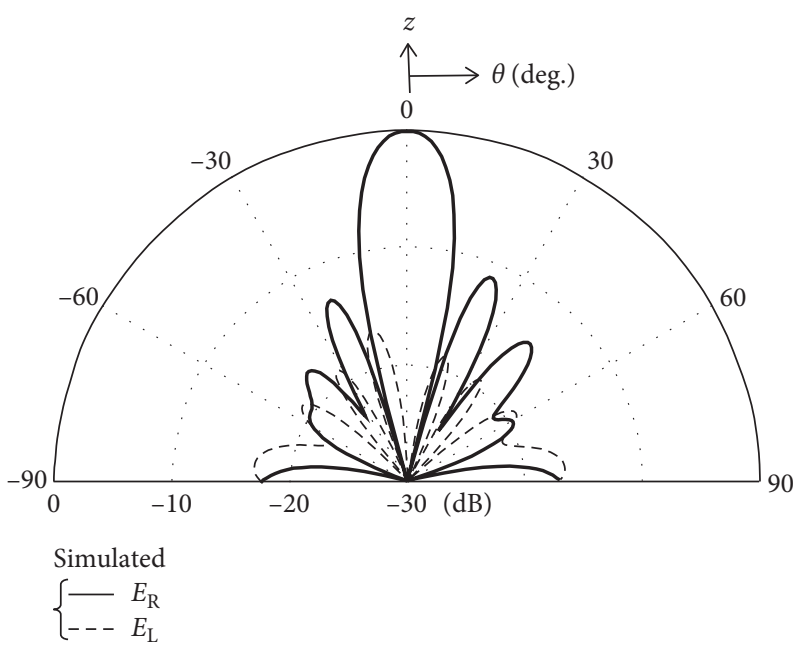

(b)

Figure 8: Radiation patterns in $\phi=0^{\circ}$ plane of line antennas with $N=4$. (a) Present line antenna with $h=0.20 \lambda_{0}$. (b) Conventional line antenna with $h=0.125 \lambda_{0}[6]$.

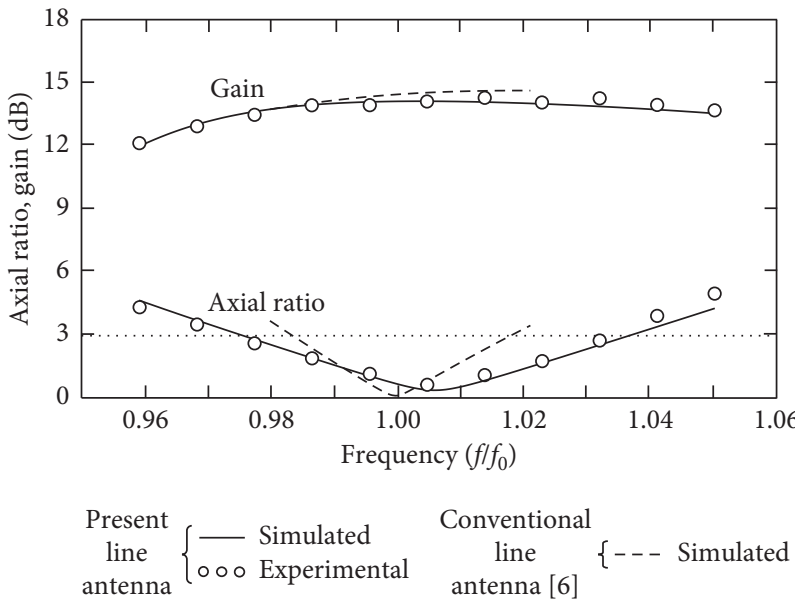

(a)

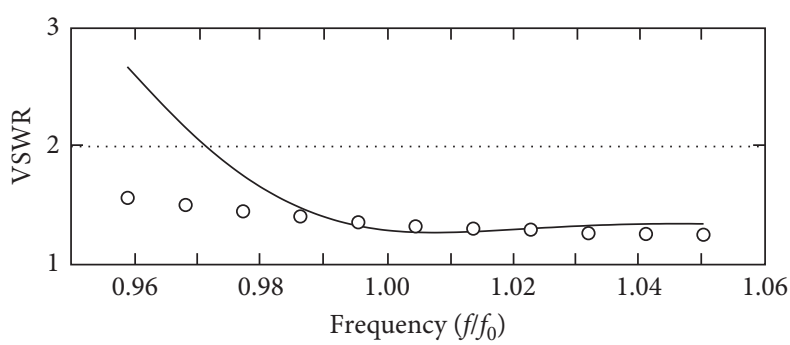

Present loop-line $\{$ - Simulated antenna $\{00$ Experimental

(b)

FIGURE 9: Frequency responses of axial ratio, gain, and VSWR of the present line antenna with $N=4$ and $h=0.20 \lambda_{0}$. (a) Axial ratio and gain, together with those of conventinal line antenna with $h=0.125 \lambda_{0}$ [6]. (b) VSWR.

one by a factor of 1.8. The solid line in Figure 9(b) shows the simulated VSWR versus frequency, which is less than 2 in the $\mathrm{CP}$ wave bandwidth.

To verify the simulated results, we perform experimental work. A loop-line antenna is fabricated at $f_{0}=3 \mathrm{GHz}$ using the ground plane of $3 \lambda_{0} \times 9 \lambda_{0}$.
Photographs of the fabricated antenna are shown in Figure 10. The dots and small circles in Figure 8(a) show the experimental results of principal $\left(E_{\mathrm{R}}\right)$ and cross-polarization $\left(E_{\mathrm{L}}\right)$ components of the radiation pattern, respectively. They agree well with the simulated results shown with solid and dotted lines. Agreement between the 


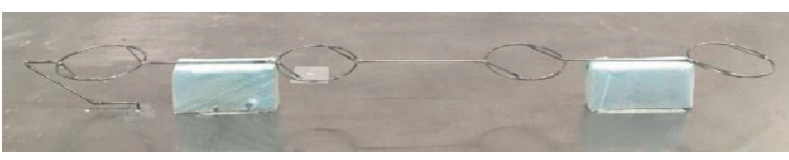

(a)

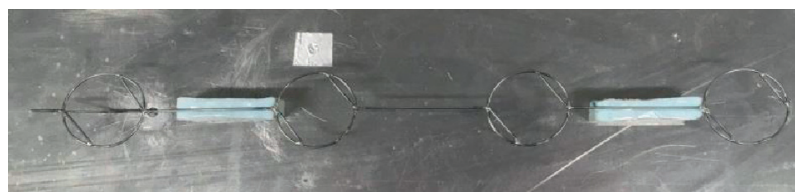

(b)

FIgURE 10: Photographs of the prototype used in experimental work. (a) Perspective view. (b) Top view.

TABle 2: Comparison with other transmission-line antennas.

\begin{tabular}{lcccc}
\hline Forms of array structure & Operating frequency & CP wave bandwidth & Radiation efficiency & Matched termination \\
\hline Rampart line [3] & $15 \mathrm{GHz}$ & - & - & Required \\
Chain line [9] & $3 \mathrm{GHz}$ & - & $50 \sim 60 \%$ & Required \\
Crank line [10] & $12 \mathrm{GHz}$ & $(3 \%)$ & - & Required \\
Loop line [11] & - & - & $\approx 100 \%$ & Required \\
Loop line (prototype) & $3 \mathrm{GHz}$ & $6 \%$ & Not required \\
\hline
\end{tabular}

(3\%): not clear which bandwidth it is for CP wave or VSWR.

experimental and simulated results is also obtained for the frequency responses of the axial ratio, gain and VSWR, as shown in Figure 9. The small circles and solid lines are experimental and simulated results, respectively.

We finally compare the results with those of line antennas investigated by other researchers. Table 2 summarizes the comparison. It is emphasized that there is no need for a matched termination in the present line antenna (prototype) [6], resulting in higher radiation efficiency. The prototype has a radiation efficiency of almost $100 \%$ since there are only conductor losses that are negligible up to a $12 \mathrm{GHz}$ band [4].

\section{Conclusions}

We have analyzed a loop antenna located at height $h$ above a ground plane. It is found that when $h<0.18 \lambda_{0}$ there exist two circularly polarized (CP) loops having different configuration values from each other and that a maximum CP wave bandwidth of $8 \%$ is obtained at $h=0.20 \lambda_{0}$. Further analysis reveals that the loop antenna has a composite configuration consisting of two CP loops, leading to wideband CP radiation. Using this composite loop as a radiation element, we design a transmission-line antenna. It is demonstrated both numerically and experimentally that the antenna radiates a broadside beam with a CP wave bandwidth of $6 \%$, which is wider than that of a conventional antenna by a factor of 2 .

\section{Data Availability}

The numerical and experimental data used to support the findings of this study are included within the article.

\section{Conflicts of Interest}

The authors declare that there are no conflicts of interest regarding the publication of this paper.

\section{Acknowledgments}

The authors would like to thank Blair Thomson for his invaluable assistance in the preparation of this manuscript.

\section{References}

[1] J. L. Volakis, Antenna Engineering Handbook, MacGrawHill, New York, NY, USA, 4th edition, 2007.

[2] J. R. James and P. S. Hall, Handbook of Microstrip Antennas, IET, Stevenage, UK, 1989.

[3] P. S. Hall, "Microstrip linear array with polarisation control," IEE Proceedings H Microwaves, Optics and Antennas, vol. 130, no. 3, pp. 215-224, 1983.

[4] H. Nakano, T. Oka, K. Hirose, and J. Yamauchi, "Analysis and measurements for improved crank-line antennas," IEEE Transactions on Antennas and Propagation, vol. 45, no. 7, pp. 1166-1172, 1997.

[5] K. Hirose, T. Shibasaki, and H. Nakano, "Fundamental study on novel loop-line antennas radiating a circularly polarized wave," IEEE Antennas and Wireless Propagation Letters, vol. 11, pp. 476-479, 2012.

[6] K. Hirose, K. Shinozaki, and H. Nakano, "A loop antenna with parallel wires for circular polarization-its application to two types of microstrip-line antennas," IEEE Antennas and Wireless Propagation Letters, vol. 14, pp. 583-586, 2015.

[7] K. Hirose, K. Okiyama, and H. Nakano, "A loop-line antenna with wideband circular polarization," in Proceedings of the 2018 IEEE International Symposium on Antennas and Propagation \& USNC/URSI National Radio Science Meeting, pp. 1933-1934, Boston, MA, USA, July 2018.

[8] R. F. Harrington, Fields Computation by Moment Methods, Macmillan, New York, NY, USA, 1968.

[9] J. Henriksson, K. Markus, and M. Tiuri, "A circularly polarized traveling-wave chain antenna," in Proceedings of the 1979 9th European Microwave Conference, pp. 174-178, Brighton, UK, September 1979.

[10] S. Nishimura, Y. Sugio, and T. Makimoto, "Crank-type circularly polarized microstrip line antenna," in Proceedings of the 1983 Antennas and Propagation Society International Symposium, pp. 162-165, Houston, TX, USA, May 1983.

[11] T. Makimoto and S. Nishimura, "Circularly polarized microstrip line antenna," US Patent 4398 199, 1983. 